\title{
Fungos microscópicos da Mata Atlântica de Paranapiacaba, São Paulo, Brasil ${ }^{1}$
}

\author{
IRACEMA HELENA SCHOENLEIN-CRUSIUS² e ADAUTO IVO MILANEZ ${ }^{2}$
}

(recebido em 02/05/96; aceito em 18/06/97)

\begin{abstract}
Microscopical fungi of the Atlantic rainforest of Paranapiacaba, São Paulo, Brazil). In the Atlantic rainforest of Paranapiacaba, state of São Paulo, 1770 occurrences of different fungal taxa were registered from July 1988 to May 1990. For stream water 319 occurrences were reported, 405 for the soil, 565 on submerged leaves of Alchornea triplinervia (Spreng.) M. Arg., and 481 on leaves placed on the soil surface. Mastigomycotina was represented by 20 taxa with 316 occurrences, Zygomycotina by 13 taxa with 266 occurrences, Deuteromycotina by 82 taxa with 1107 occurrences, and Ascomycotina by eight taxa with 81 records. The fungi were isolated by the leaf disk washing method, soil plate method, and baiting with chitinic, cheratinic and cellulosic substrates. The decomposing leaves of A. triplinervia supported the highest number of fungal taxa and total occurrences, followed by the soil and stream water.
\end{abstract}

RESUMO - (Fungos microscópicos da Mata Atlântica de Paranapiacaba, São Paulo, Brasil). Na Mata Atlântica de Paranapiacaba, no estado de São Paulo, 1770 ocorrências de diferentes táxons de fungos foram registradas de julho de 1988 a maio de 1990. Para a água do riacho foram reportadas 319 ocorrências, 405 para o solo, 565 em folhas submersas de Alchornea triplinervia (Spreng.) M. Arg. e 481 em folhas dispostas sobre o solo. Mastigomycotina representou 316 ocorrências com 20 espécies, Zygomycotina 266 ocorrências com 13 espécies, Deuteromycotina 1107 ocorrências com 82 táxons e Ascomycotina 81 ocorrências com oito espécies. Os fungos foram isolados pelos métodos da lavagem de discos de folhas, placa de solo e iscagem utilizando substratos quitínicos, queratínicos e celulósicos. As folhas de A. triplinervia suportaram o maior número de táxons e de ocorrências de fungos, seguidas pelo solo e pela água do riacho.

Key words - Fungi, Atlantic rainforest, diversity

\section{Introdução}

Na Reserva Biológica de Paranapiacaba, SP, foram realizados diversos estudos sobre fungos, porém têm sido poucos os resultados publicados. Entre os principais estudos conduzidos na região, que efetivamente contribuíram para o conhecimento da diversidade da micota nativa, destacam-se aqueles relativos aos fungos micorrízicos do solo (Trufem \&Viriato 1990) e aos Hyphomycetes associados a folhas de Alchornea triplinervia (Spreng.) M. Arg. e de Euterpe edulis Mart. (Grandi 1993). Antunes et al. (1993) constataram o rápido poder de recuperação da diversidade dos fungos do solo da Mata Atlântica de Paranapiacaba, acidentalmente submetido ao fogo.

No ambiente aquático foram estudados os fungos zoospóricos (Rogers et al. 1970) e os Hyphomycetes (Schoenlein-Crusius \& Milanez 1990 a,b). Posteriormente, Schoenlein-Crusius et al. (1992) compararam a micota zoospórica e a de Hyphomycetes aquáticos em folhas submersas de Alchornea triplinervia, Ficus microcarpa e Quercus robur, sendo abordados aspectos qualitativos da diversidade dos

1. Parte da tese de doutorado de I. H. Schoenlein-Crusius.

2. Instituto de Botânica, Caixa Postal 4005, 01061-970 São Paulo, SP, Brasil. fungos. Mais recentemente, Pires-Zottarelli et al. (1993) apresentaram método para estimar o potencial de colonização de fungos decompositores.

O objetivo do presente artigo é apresentar metodologia e dados globais sobre a distribuição dos fungos, em termos de ocorrências e grupos taxonômicos registrados para os substratos utilizados nos diferentes ambientes estudados na Reserva Biológicade Paranapiacaba (SP).

\section{Material e métodos}

A Reserva Biológica de Paranapiacaba, SP, localizada no município de Santo André, a $23^{\circ} 46^{\prime} \mathrm{S}, 46^{\circ} 18^{\prime} \mathrm{W}$ e $750-890 \mathrm{~m}$ de altitude, possui 336 ha e está sob a responsabilidade do Instituto de Botânica. Apresenta clima do tipo Cfb segundo o sistema de Köppen, com precipitações anuais em torno de $3400 \mathrm{~mm}$ e umidade relativa próxima a $100 \%$. Segundo Setzer (1966) a temperatura média nos meses mais frios (junho/julho) é inferior a $18^{\circ} \mathrm{C}$ e permanece abaixo de $22^{\circ} \mathrm{C}$ nos meses mais quentes (janeiro/ fevereiro). O elevado índice de precipitação durante todo o ano caracteriza o clima como super-úmido, sem estações secas (Funari et al. 1986).

No presente trabalho considerou-se Mata Atlântica a vegetação que se desenvolveu sobre toda a cadeia montanhosa justamarítima e de planalto, que se estende desde o Rio Grande do Sul até o extremo nordeste (Rizzini 1979). Descrições detalhadas sobre as características da vegetação são apresentadas por Coutinho (1962) e De Vuono et al. (1989).

Para estudar os fungos no ambiente aquático, foi escolhido um dos riachos que percorre toda a Reserva, sendo que o trecho amostrado apresenta distância aproximada de 
$4 \mathrm{~m}$ entre as margens, profundidade média de 80 a $100 \mathrm{~cm}$ na época chuvosa (dezembro a fevereiro) e de 30 a $50 \mathrm{~cm}$ na época de menores índices pluviométricos (junho a agosto). O leito é arenoso e macio. A água apresenta-se transparente, porém não é potável, apresentando em torno de 300 bactérias coliformes fecais por $100 \mathrm{ml}$ de amostra, segundo exames realizados pelo Instituto Adolfo Lutz e CETESB, em maio de 1988 e de 1989. No presente trabalho foram escolhidos cinco pontos de coleta ao longo do riacho, com distância de aproximadamente $50 \mathrm{~m}$ entre eles, demarcados por estacas.

Também foram escolhidas cinco áreas de $50 \mathrm{~m}^{2}$ próximas ao riacho para o estudo dos fungos no ambiente terrestre.

Em junho de 1988 e de 1989, folhas recém-caídas de Alchornea triplinervia (Spreng.) M. Arg., uma das espécies mais abundantes na Reserva, foram coletadas em peneiras de tela de náilon e em sacos plásticos abertos, estendidos e amarrados entre as árvores. Estas folhas foram triadas, divididas em amostras de $20 \mathrm{~g}$ e confinadas em 400 sacos de tela de náilon com $20 \mathrm{~cm}$ de comprimento, $20 \mathrm{~cm}$ de largura e malha com $1 \mathrm{~mm}$ de diâmetro. Duzentos sacos foram distribuídos nos cinco pontos de coleta ao longo do riacho, sendo que 40 unidades foram amarradas na base de cada estaca. Procedimento semelhante foi conduzido no ambiente terrestre, distribuindo-se 40 sacos contendo folhas sobre a superfície de cada área pré-definida.

As coletas mensais de 15 sacos contendo folhas do ambiente terrestre, 15 sacos contendo folhas submersas, cinco amostras compostas de solo (aproximadamente $300 \mathrm{~g}$ de cada área demarcada) e cinco amostras compostas de água do riacho (aproximadamente $250 \mathrm{ml}$ de cada ponto) foram realizadas em dois períodos, sendo o primeiro de julho de 1988 a junho de1989 e a repetição, de julho de 1989 a maio de 1990 .

As folhas submersas e as dispostas sobre o solo foram coletadas mensalmente e tratadas pela técnica de lavagem de discos de folhas (Pugh et al. 1972), que consiste em lavar os discos com diâmetro de $5 \mathrm{~mm}$ (500 unidades no presente estudo), com água destilada estéril $(50 \mathrm{ml})$ sucessivamente por 30 vezes, eliminando propágulos aderidos à superfície foliar. Após a lavagem, os discos foram enxutos em folhas de papel de filtro previamente esterilizadas e dispostos de modo eqüidistante em 20 placas de Petri (cada uma com cinco discos), onde foram colocados um dos seguintes meios de cultura: batatadextrose-ágar (Difco Manual 1972), amido-ágar (Coelho \&Drozdowicz 1976 in Costa 1983), celulose-ágar (Eggins \& Pugh 1962) e pectinaágar (Difco Manual 1972), perfazendo 80 placas de Petri contendo discos de folhas submersas e 80 placas contendo discos de folhas coletadas do solo.

Vinte discos de folhas também foram incubados em água destilada estéril contendo iscas para fungos quitinofílicos (iscas de ecdise de cobra e exoesqueleto de camarão), queratinofílicos (iscas de fios de cabelo loiro) e celulolíticos (iscas de palha de milho e celofane). Para fungos zoospóricos que apresentam afinidade por substâncias de reserva foram utilizados grãos de pólen de Pinus sp. e para o isolamento dos Oomycetes (Mastigomycotina) os discos de folhas foram colocados em contato com sementes de sorgo, partidas ao meio, previamente fervidas (Milanez 1984). Foram preparadas cinco placas de Petri contendo as iscas com os discos de folhas coletadas do solo e cinco com os discos de folhas submersas.

Alíquotas de $1 \mathrm{ml}$ das amostras compostas de água do riacho foram distribuídas em 40 placas de Petri, dez unidades para cada um dos meios de cultura utilizados para o isolamento dos fungos das folhas, adaptando-se analogamente a técnica de Warcup (1950). Também foram preparadas cinco placas de Petri contendo $10 \mathrm{ml}$ de cada uma das amostras de água do riacho, adicionando-se as iscas quitínicas, queratínicas e celulósicas para isolar os fungos zoospóricos (Mastigomycotina)

Para isolar os fungos das cinco amostras compostas de solo foi utilizado o método da placa de solo (Warcup 1950), modificado pela diluição das alíquotas. Foram preparadas 40 placas de solo utilizando-se os mesmos meios de cultura empregados para as folhas submersas e as dispostas sobre o solo, bem como cinco placas de Petri, cada uma contendo $1 \mathrm{~g}$ de amostra composta, aproximadamente $10 \mathrm{ml}$ de água destilada estéril e o conjunto de substratos quitínicos, queratínicos e celulósicos para o isolamento de fungos zoospóricos.

Após sete dias à temperatura ambiente $\left(20\right.$ a $\left.25^{\circ} \mathrm{C}\right)$, as colônias que se desenvolveram ao redor dos discos de folhas, nas placas de solo e nas das alíquotas de água, foram transferidas para novo meio de cultura. As iscas começaram a ser observadas ao microscópio óptico após o quinto dia de crescimento. Os substratos colonizados por fungos zoospóricos foram transferidos para placas de Petri contendo água destilada estéril e novos substratos.

As identificações dos fungos foram realizadas com o apoio da bibliografia corrente para Mastigomycotina, Zygomycotina, Ascomycotina e Deuteromycotina mencionada em SchoenleinCrusius (1993).

Após as identificações, as colônias foram repicadas em tubos contendo meios de cultura específicos para cada grupo e armazenados em geladeira a $4^{\circ} \mathrm{C}$ na Coleção de Culturas da Seção de Micologia e Liquenologia do Instituto de Botânica.

As ocorrências apresentadas referem-se a presença do fungo no substrato, independentemente do número de colônias observadas do mesmo, durante a fase de isolamento.

Com base nas ocorrências totais dos fungos nos diferentes ambientes e substratos, foi calculado o índice de similaridade de Morisita (Brown \& Zar 1984).

\section{Resultados e Discussão}

O experimento conduzido na Reserva Biológica de Paranapiacaba resultou no registro de 1770 ocorrências de fungos (tabela 1). As folhas submersas apresentaram maior número de ocorrências, seguido pelas folhas do ambiente terrestre, o solo e a água do riacho. O número total de táxons isolados apresentou-se maior nas folhas submersas, seguido pelas folhas colocadas sobre o solo, água e solo, o que sugere que as folhas, especialmente as submersas, ofereceram condições favoráveis para a colonização por fungos. Este resultado ressalta a importância das mesmas como fonte de nutrientes e inóculo para o ambiente aquático (Barlocher \& Kendrick 1974).

Do número total de ocorrências, 1107 corresponderam a Deuteromycotina, 266 a Zygomycotina, 316 a Mastigomycotina (incluindo Chytridiomycetes e Oomycetes) e 81 a Ascomycotina. O número total de táxons foi 123 , sendo 82 pertencentes a Deuteromycotina, 13 a Zygomycotina, 20 a Mastigomycotina (incluindo Chytridiomycetes e Oomycetes) e oito a Ascomycotina (tabela 1). 
Tabela 1. Ocorrência de fungos isolados da água (A), solo (S) e folhas de Alchornea triplinervia colocadas no ambiente aquático (FA) e no terrestre (FS) na Reserva Biológica de Paranapiacaba, SP, de julho de 1988 a maio de 1990.

\begin{tabular}{|c|c|c|c|c|c|}
\hline \multirow{2}{*}{ Deuteromycotina } & \multicolumn{5}{|c|}{ Ambientes } \\
\hline & A & $\mathrm{S}$ & FA & FS & Total \\
\hline Acremonium fusidioides (Nicot) W. Gams & 0 & 2 & 0 & 0 & 2 \\
\hline Acremonium strictum $\mathrm{W}$. Gams & 3 & 5 & 9 & 4 & 21 \\
\hline Alternaria alternata (Fr.) Keissler & 0 & 6 & 10 & 10 & 26 \\
\hline Anguillospora crassa Ingold & 6 & 0 & 7 & 0 & 13 \\
\hline Aspergillus clavatus Dezm. & 2 & 11 & 13 & 6 & 32 \\
\hline Aspergillus flavus Link. & 0 & 4 & 0 & 0 & 4 \\
\hline Aspergillus fumigatus Fries & 1 & 0 & 0 & 0 & \\
\hline Aspergillus glaucus (Mich. ex L.: Fr.) Link. & 0 & 13 & 0 & 6 & 19 \\
\hline Aspergillus niger van Thieghem & 7 & 12 & 11 & 4 & 34 \\
\hline Aspergillus alutaceus Berk \& Curt. (= A. ochraceus) & 2 & 0 & 5 & 0 & 7 \\
\hline Aspergillus ornatus Raper \& Fennell & 0 & 5 & 0 & 0 & 5 \\
\hline Aspergillus sydowii Bain \& Sort. & 0 & 0 & 0 & 6 & 6 \\
\hline Aspergillius ustus (Bain) Thom \& Church & 4 & 0 & 0 & 6 & 10 \\
\hline Aspergillus versicolor (Vuill.) Tiraboshi & 0 & 0 & 0 & 5 & 5 \\
\hline Cladosporium cladosporioides (Fresen) De Vries & 10 & 7 & 9 & 0 & 26 \\
\hline Cladosporium herbarum (Pers) Link. ex S. F. Gray & 0 & 0 & 7 & 0 & 7 \\
\hline Cladosporium oxysporum Berk \& Curt. & 8 & 7 & 8 & 6 & 29 \\
\hline Cladosporium sphaerospermum Penz. & 5 & 2 & 8 & 3 & 18 \\
\hline Colletotrichum orbiculare (Beak. \& Mont.) Arx & 0 & 0 & 2 & 0 & 2 \\
\hline Curvularia brachyspora Boedijn & 1 & 0 & 4 & 0 & 5 \\
\hline Curvularia lunata (Wakker) Boedijn & 0 & 0 & 0 & 3 & 3 \\
\hline Cylindrocladium scoparium Morgan & 0 & 6 & 14 & 5 & 25 \\
\hline Diplococcium sp. & 0 & 0 & 0 & 1 & 1 \\
\hline Epicoccum purpurescens Ehrenb. ex Schlecht & 5 & 4 & 10 & 8 & 27 \\
\hline Fusarium antophylum (A. Braun) Wollenw. & 3 & 0 & 0 & 0 & 3 \\
\hline Fusarium avenaceum (Fr.) Sacc. & 0 & 4 & 0 & 0 & 4 \\
\hline Fusarium clamidosporium Wollenw. \& Reinking & 0 & 0 & 5 & 7 & 12 \\
\hline Fusarium graminearum Schwabe & 5 & 3 & 8 & 16 & 32 \\
\hline Fusarium graminum Corda & 0 & 0 & 0 & 1 & 1 \\
\hline Fusarium heterosporum Nees & 0 & 1 & 2 & 1 & 4 \\
\hline Fusarium lateritium Nees & 0 & 0 & 8 & 11 & 19 \\
\hline Fusarium moniliforme Sheldon & 6 & 0 & 1 & 2 & 9 \\
\hline Fusarium nivale (Fr.) Ces. & 6 & 0 & 5 & 6 & 17 \\
\hline Fusarium oxysporum Schlecht. emend. Snyd. \& Hans. & 10 & 23 & 23 & 14 & 70 \\
\hline Fusarium poae (Peck) Wollenw. & 8 & 5 & 2 & 5 & 20 \\
\hline Fusarium sambucinum Fuckel & 0 & 0 & 1 & 3 & 4 \\
\hline \multicolumn{6}{|l|}{ Fusarium solani (Mart.) Appel \& Wollenw. emend. } \\
\hline Snyd. \& Hans. & 7 & 11 & 17 & 14 & 49 \\
\hline Fusarium sporotrichioides Sherb. & 0 & 6 & 0 & 5 & 11 \\
\hline Fusarium tricinctum (Corda) Sacc. & 0 & 0 & 0 & 5 & 5 \\
\hline Fusarium ventricosum Appel \& Wollenw. & 0 & 0 & 0 & 6 & 6 \\
\hline Geotrichum candidum Link ex Leman & 2 & 13 & 0 & 0 & 15 \\
\hline Gliocladium roseum Bainier & 4 & 6 & 6 & 8 & 24 \\
\hline \multicolumn{6}{|l|}{ Gliomastix murorum (Corda) Hughes v.felina } \\
\hline (Marchal) Hughes & 0 & 5 & 0 & 0 & 5 \\
\hline Graphium sp. & 0 & 0 & 0 & 2 & 2 \\
\hline Insecticola sp. & 0 & 0 & 0 & 5 & 5 \\
\hline Lunulospora curvula Ingold & 6 & 0 & 8 & 0 & 14 \\
\hline Nigrospora oryzae Berk. \& Br. & 2 & 2 & 5 & 1 & 10 \\
\hline Oedocephalum sp. & 0 & 0 & 0 & 1 & 1 \\
\hline
\end{tabular}


(cont.)

\begin{tabular}{|c|c|c|c|c|c|}
\hline \multirow{2}{*}{ Deuteromycotina } & \multicolumn{5}{|c|}{ Ambientes } \\
\hline & A & S & FA & FS & Total \\
\hline \multicolumn{6}{|l|}{ Paecilomyces carneus (Duché \& Heim) } \\
\hline A. H. S. Brown \& G. Sam. & 2 & 5 & 0 & 0 & 7 \\
\hline Paecilomyces clavisporus Hammill & 3 & 0 & 2 & 4 & 9 \\
\hline Paecilomyces javanicus (Friederichs \& Bally) Brown \& Smith & 5 & 6 & 7 & 7 & 25 \\
\hline Paecilomyces variotii Bain & 0 & 2 & 6 & 0 & 8 \\
\hline Penicillium aurantiobrunneum Dierckx & 0 & 0 & 1 & 0 & 1 \\
\hline Penicillium brevicompactum Dierckx & 4 & 0 & 1 & 0 & 5 \\
\hline Penicillium chrysogenum Thom & 1 & 0 & 0 & 0 & 1 \\
\hline Penicillium corilophlylum Dierckx & 0 & 0 & 1 & 0 & 1 \\
\hline Penicillium fellutanum Biourge & 2 & 0 & 0 & 0 & 2 \\
\hline Penicillium hirsutum Dierckx & 17 & 9 & 21 & 19 & 66 \\
\hline Penicillium janthinelum Biourge & 0 & 0 & 0 & 2 & 2 \\
\hline Penicillium mineoluteum Dierckx & 3 & 0 & 0 & 0 & 3 \\
\hline Penicillium oxalicum Currie \& Thom & 3 & 0 & 1 & 0 & 4 \\
\hline Penicillium pinophyllum Hedgecock & 1 & 0 & 0 & 0 & 1 \\
\hline Penicillium variabile Sopp. & 1 & 4 & 0 & 0 & 5 \\
\hline Penicillium viridicatum Westling & 0 & 0 & 0 & 5 & 5 \\
\hline Penicilliuin waksmanii Zaleski & 2 & 9 & 0 & 0 & 11 \\
\hline Periconia atropurpurea (Berk \& Curt.) Litvinov & 0 & 0 & 0 & 1 & 1 \\
\hline Pestalotia clavata Cooke \& Ell. & 7 & 0 & 9 & 11 & 27 \\
\hline Phoma chrysantemicola Hollos. & 0 & 0 & 6 & 0 & 6 \\
\hline Phoma tropica Schneider \& Boerema & 2 & 0 & 0 & 0 & 2 \\
\hline Phoma jolyana Pirozynski \& Morgan-Jones & 0 & 0 & 1 & 0 & 1 \\
\hline Stilbella sp. & 0 & 0 & 0 & 1 & 1 \\
\hline Tetrachaetum elegans Ingold & 0 & 0 & 13 & 0 & 13 \\
\hline Trichoderma hamatum (Bon.) Bain & 0 & 1 & 2 & 0 & 3 \\
\hline Trichoderma harzianum Riffai & 2 & 0 & 2 & 8 & 12 \\
\hline Trichoderma koningii Oud. & 5 & 9 & 13 & 16 & 43 \\
\hline Trichoderma longibrachiatum Riffai & 0 & 1 & 1 & 5 & 7 \\
\hline Trichoderma pseudokoningii Riffai & 5 & 2 & 6 & 8 & 21 \\
\hline Trichoderma viride Pers. ex Gray & 23 & 19 & 23 & 20 & 85 \\
\hline Tripospermum sp. & 0 & 0 & 9 & 0 & 9 \\
\hline Triscelophorus monosporus Ingold & 0 & 0 & 14 & 0 & 14 \\
\hline Verticillium fungicola (Preuss) Hasselr. & 3 & 2 & 8 & 6 & 19 \\
\hline Verticillium lecanii (Zimm.) Viegas & 0 & 4 & 6 & 7 & 17 \\
\hline Total de ocorrências & 204 & 236 & 361 & 305 & 1107 \\
\hline Total de táxons & 44 & 38 & 49 & 48 & 82 \\
\hline Zygomycotina & A & $\mathrm{S}$ & FA & FS & Total \\
\hline Absidia cylindrospora Hagem & 0 & 12 & 0 & 7 & 19 \\
\hline Mucor circinelloides van Thieghem & 3 & 9 & 5 & 8 & 25 \\
\hline Mucor circinelloides van Thieghem f. janssenii (Lendner) Schipper & 0 & 0 & 6 & 11 & 17 \\
\hline Mucor genevensis Wehmer & 0 & 0 & 0 & 8 & 8 \\
\hline Mucor hiemalis Wehmer & 20 & 23 & 16 & 19 & 78 \\
\hline Mucor hiemalis Wehmer f. silvaticus (Hagem) Schipper & 0 & 0 & 7 & 12 & 19 \\
\hline Mucor racemosus Fres. f. sphaerosporus (Hagem) Schipper & 0 & 0 & 0 & 7 & 7 \\
\hline Rhizopus arrhizus Fischer & 2 & 17 & 6 & 5 & 30 \\
\hline Rhizopus oligosporus Fischer & 0 & 0 & 0 & 5 & 5 \\
\hline
\end{tabular}


(cont.)

\begin{tabular}{|c|c|c|c|c|c|}
\hline \multirow{2}{*}{ Zygomycotina } & \multicolumn{5}{|c|}{ Ambientes } \\
\hline & A & $\mathrm{S}$ & FA & FS & Total \\
\hline Rhizopus oryzae (Went. \& Prinsen) Geerlings & 0 & 12 & 5 & 4 & 21 \\
\hline Zygorrhynchus japonicus Viull. & 0 & 0 & 0 & 6 & 6 \\
\hline Zygorrhynchus macrocarpus Ling-Young & 0 & 6 & 0 & 4 & 10 \\
\hline Zygorrhynchus moelleri Viull. & 1 & 11 & 3 & 6 & 21 \\
\hline Total de ocorrências & 26 & 90 & 48 & 102 & 266 \\
\hline Total de táxons & 04 & 07 & 07 & 13 & 13 \\
\hline Mastigomycotina & A & $\mathrm{S}$ & FA & FS & Total \\
\hline Achlya dubia Coker & 6 & 5 & 6 & 0 & 17 \\
\hline Achlya flagellata Coker & 4 & 2 & 6 & 3 & 15 \\
\hline Achlya radiosa Maurizio & 4 & 1 & 12 & 0 & 17 \\
\hline Dictyuchus sp. & 0 & 0 & 7 & 0 & 7 \\
\hline Pythium (estéril) & 2 & 7 & 6 & 5 & 20 \\
\hline Saprolegnia ferax (Gruith.) Thuret. & 6 & 6 & 8 & 0 & 20 \\
\hline Saprolegnia parasitica Coker & 0 & 0 & 6 & 4 & 10 \\
\hline Catenophlyctis variabilis (Karling) Karling & 8 & 0 & 7 & 0 & 15 \\
\hline Cladochytrium replicatum Karling & 4 & 2 & 7 & 5 & 18 \\
\hline Karlingia rosea (De Bary \& Woronin) Johanson & 5 & 10 & 7 & 13 & 35 \\
\hline Karlingiomyces sp. & 0 & 0 & 3 & 0 & 3 \\
\hline Nowakowskiella elegans (Nowak.) Schroeter & 8 & 7 & 10 & 7 & 32 \\
\hline Phlyctochytrium sp. & 2 & 0 & 3 & 0 & 5 \\
\hline Polychytrium aggregatum Ajello & 9 & 5 & 14 & 4 & 32 \\
\hline Rhizidium chitinophylum Antikajian & 0 & 1 & 4 & 0 & 5 \\
\hline Rhizophlyctis sp. & 0 & 0 & 7 & 0 & 7 \\
\hline Rhizophydium chitinophyllum Sparrow & 2 & 0 & 3 & 0 & 5 \\
\hline Rhizophydium elyensis Sparrow & 10 & 10 & 8 & 6 & 34 \\
\hline Rhizophydium sphaerotheca Zopf & 1 & 0 & 5 & 0 & 6 \\
\hline Rhizophydium stipitatum Sparrow & 4 & 3 & 4 & 2 & 13 \\
\hline Total de ocorrências & 75 & 59 & 133 & 49 & 316 \\
\hline Total de táxons & 16 & 12 & 12 & 09 & 20 \\
\hline Ascomycotina & A & $\mathrm{S}$ & FA & FS & Total \\
\hline Chaetomium globosum Kunze ex Steud. & 2 & 15 & 4 & 6 & 27 \\
\hline Eupenicillium javanicum (van Beyma) Stolk. \& Scott & 10 & 0 & 0 & 0 & 10 \\
\hline Gelasinospora cerealis Dowding & 0 & 0 & 5 & 6 & 11 \\
\hline Gelasinospora retispora Cain. & 0 & 0 & 0 & 5 & 5 \\
\hline Microsphaeriopsis olivacea (Bonord.) Hohn. & 0 & 5 & 6 & 0 & 11 \\
\hline Nectria haematococca Beak. \& Br. & 0 & 0 & 7 & 8 & 15 \\
\hline Talaromyces flavus (Klocker) Stolk. \& Samson & 0 & 0 & 1 & 0 & 1 \\
\hline Thielavia basicola Zopf & 1 & 0 & 0 & 0 & 1 \\
\hline Total de ocorrências & 13 & 20 & 23 & 25 & 81 \\
\hline Total de táxons & 02 & 02 & 06 & 04 & 08 \\
\hline Total geral de ocorrências & 319 & 405 & 565 & 481 & 1770 \\
\hline Total geral de táxons & 65 & 59 & 81 & 74 & 123 \\
\hline
\end{tabular}


O predomínio de Deuteromycotina em termos de diversidade e ocorrência tem sido freqüentemente reportado em diversos ecossistemas, como a mata de planalto do Parque Estadual das Fontes do Ipiranga, São Paulo (Schoenlein-Crusius \& Milanez 1989, Ninomiya et al. 1993), a mata da região de Itapecerica da Serra (Schoenlein-Crusius et al. 1990) e a Mata Atlântica da Reserva de Juréia-Itatins, São Paulo (Attili 1994, Garlipp 1995). Incluídos em Deuteromycotina, a presença de diversos Hyphomycetes tem sido observada sobre folhas em decomposição de Cedrella fissilis Vell. em Maringá, PR (Grandi \& Gusmão, 1995) e em mata secundária em São Paulo (Grandi et al. 1995).

O predomínio de Deuteromycotina em substratos em decomposição no ambiente terrestre foi verificado em folhedo na mata pluvial em Pernambuco (Maia 1983) e na sucessão fúngica em folhas de Ocotea pulchella (Nees) Mez no cerrado de Corumbataí (Schoenlein-Crusius \& Tauk 1991), em que mais de $80 \%$ da micota constituiu-se de gêneros de Hyphomycetes.

Os resultados obtidos estão de acordo com os de Attili (1994) que isolou 67 táxons de fungos celulolíticos do solo, da Reserva Ecológica Juréia-Itatins, sendo 55 de Deuteromycotina, dez de Ascomycotina e dois de Zygomycotina. Na mesma região, Garlipp (1995) isolou 63 táxons, distribuídos em 59 de Deuteromycotina, três de Zygomycotina e um representante de Ascomycotina, de amostras de solo.

Antunes et al. (1993) isolaram 32 táxons, distribuídos em 12 de Deuterormycotina, dez de Zygomycotina, dois de Ascomycotina e oito de Mastigomycotina do solo na Mata Atlântica de Paranapiacaba, enquanto Ninomiya et al. (1993) obtiveram 30 táxons distribuídos em 22 de Deuteromycotina, sete de Mastigomycotina e um de Ascomycotina no solo da mata de planalto do Parque Estadual das Fontes do lpiranga.

Os resultados encontrados para as folhas de $A$. triplinervia submersas (tabela 1) são comparáveis aos observados em folhas de Ficus microcarpa L. f. submersas em um lago no Parque Estadual das Fontes do lpiranga, com o isolamento de 30 táxons, sendo 16 fungos imperfeitos terrestres, quatro tetrarradiados (Deuteromycotina), oito fungos zoospóricos (Mastigomycotina) e duas espécies de Zygomycotina (Schoenlein-Crusius \& Milanez 1989). Em folhas de Quercus robur L. foram encontrados 36 táxons, sendo 17 Hyphomycetes terrestres, oito Hyphomycetes aquáticos (Deuteromycotina), três espécies de Zygomycotina e oito fungos zoospóricos (Mastigomycotina) (Schoenlein-Crusius et al. 1990).

Nas folhas de A. triplinervia colocadas sobre o solo foram encontradas 305 ocorrências de Deuteromycotina, 102 de Zygomycotina, 49 de Mastigomycotina e 25 de Ascomycotina. Foram isolados 74 táxons sendo 48 de Deuteromycotina, 13 de Zygomycotina, nove de fungos zoospóricos e quatro Ascomycotina (tabela 1). Grandi (1993) obteve 37 táxons de Hyphomycetes sobre folhas de A. triplinervia e E. edulis na mesma região. O número de ocorrências e de táxons de fungos imperfeitos (Deuteromycotina) encontrados no presente estudo pode ter sido maior devido ao uso de metodologias que permitem o desenvolvimento de fungos esporulantes sobre os meios de cultura, o que normalmente se traduz na ampliação da diversidade da micota obtida.

Segundo o índice de Morisita o maior índice de similaridade (83\%) foi encontrado entre a micota da água e a das folhas submersas, enquanto o menor índice (74\%) foi encontrado entre a micota da água e a do solo. As micotas das folhas colocadas no solo e as submersas apresentaram similaridade de $81 \%$. A micota aquática e a das folhas colocadas sobre o solo apresentaram 76\% de similaridade. A micota do solo apresentou $75 \%$ de similaridade em relação à micota das folhas colocadas no ambiente terrestre e $76 \%$ de semelhança com a micota das folhas submersas no riacho. Segundo Christensen (1989), índices de similaridade em torno de $70 \%$ podem ser considerados elevados, indicando proximidade entre as populações fúngicas. No entanto, considera-se que ainda há falta de dados cumulativos para as regiões de clima tropical, que possam indicar o significado dos índices de similaridade encontrados. A diversidade dos fungos em Paranapiacaba pode ser considerada elevada, o que confirma, pelo menos em parte, a adequação da metodologia utilizada para estudos de biodiversidade da micota nativa.

\section{Referências bibliográficas}

ANTUNES, M.F.R., NINOMIYA, A. \& SCHOENLEINCRUSIUS, I.H. 1993. Efeitos de queimada sobre a micota de solo na mata atlântica na Reserva Biológica do Alto da Serra de Paranapiacaba, SP. Hoehnea 19:1-8. 
ATTILI, D.S. 1994. Isolamento, identificação e ecologia de fungos celulolíticos do solo da Estação Ecológica JuréiaItatins, SP. Tese de doutorado, Universidade Estadual Paulista, Rio Claro.

BARLOCHER, F. \& KENDRICK, B. 1974. Dynamics of the fungal population on leaves in the stream. J. Ecol. 62: 761-791.

BROWN, J. E. \& ZAR, J. H. 1984. Field and laboratories methods for general Ecology. Brown Publishing, Des Moines, Iowa.

CHRISTENSEN, M. 1989. A view of fungal ecology. Mycologia 81:1-19.

COSTA, S.G. 1983. Efeito da adição de vinhaça na dinâmica da microflora do solo de cerrado de Corumbataí, SP. Dissertação de mestrado, Universidade Estadual Paulista, Rio Claro.

COUTINHO, L.M. 1962. Contribuição ao conhecimento da ecologia da mata pluvial tropical. Bol. Fac. Fil. Ciên. Let. USP 18:11-219.

DE VUONO, Y.S., DOMINGOS, M. \& LOPES, M.J.M.S. 1989. Decomposição da serapilheira e liberação de nutrientes na floresta da Reserva Biológica de Paranapiacaba, SP, sujeita aos poluentes atmosféricos de Cubatão, São Paulo, Brasil. Hoehnea 16:179-193.

DIFCO MANUAL. 1972. Difco Laboratories, Michigan.

EGGINS, H. O. W. \& PUGH, G. J. F. 1962. Isolation of cellulose decomposing fungi from the soil. Nature 193:94-95.

FUNARI, F.L., DE VUONO, Y.S. \& SALUM, S. 1986. Balanço hídrico de duas áreas da mata atlântica: Reserva Biológica do Alto da Serra de Paranapiacaba e Parque Estadual da Ilha do Cardoso, SP. In Anais do 6을 Congresso da Sociedade Botânica de São Paulo, São Paulo, p. 95.

GARLIPP, A.B. 1995. Isolamento e identificação de fungos filamentosos do solo do Banhado Grande na Estação Ecológica de Juréia-Itatins. Dissertação de mestrado, Universidade Estadual Paulista, Rio Claro.

GRANDI, R.A.P. 1993. Hyphomycetes associados a folhas em decomposição de Alchornea triplinervia (Spreng.) M. Arg. e Euterpe edulis Mart. Tese de doutorado, Universidade de São Paulo, São Paulo.

GRANDI, R.A.P. \& GUSMÃO, L.F. 1995. Espécies de Gyrothrix (Hyphomycetes) no folhedo de Cedrella fissilis Vell., em Maringá, PR, Brasil. Hoehnea 22:191-196.

GRANDI, R.A.P., GRANDI, A.C. \& DELITTI, W.B.C. 1995. Hyphomycetes sobre folhas em decomposição de Cedrella fissilis Veil. Hoehnea 22:27-37.

MAIA, L. C. 1983. Sucessão de fungos em folhedo de floresta tropical úmida. Editora da Universidade Federal de Pernambuco, Recife.

MILANEZ, A.I. 1984. Fungos zoospóricos do Estado de São Paulo. II. Chytridiomycetes da região oeste. Rickia 11:115-127.

NINOMIYA, A., ANTUNES, M.F.R. \& SCHOENLEINCRUSIUS, I.H. 1993. Fungi from soil affected by birds in the "Parque Estadual das Fontes do Ipiranga", São Paulo State, Brazil. Rev. Microbiol. 24:149-151.
PIRES-ZOTTARELLI, C. L. A., SCHOENLEIN-CRUSIUS, I.H. \& MILANEZ, A.I. 1993. Quantitative estimation of zoosporic fungi and aquatic hyphomycetes on leaves submerged in a stream in the atlantic rainforest, in the state of São Paulo, Brazil. Rev. Microbiol. 24:192-197.

PUGH, G.J.F., BUCKEY, N.G. \& MULDER, J. 1972. The role of phylloplane fungi in the early colonization of leaves. Symp. Biol. Hung. 11:329-333.

RIZZINI, C.T. 1979. Tratado de Fitogeografia do Brasil. Editora da Universidade de São Paulo, São Paulo.

ROGERS, A.L., MILANEZ, A.I. \& BENEKE, E.S. 1970. Additional aquatic fungi from São Paulo State. Rickia 5:93-110.

SCHOENLEIN-CRUSIUS, I.H. 1993. Sucessão fúngica em folhas de Alchornea triplinervia (Spreng.) M. Arg. em ambientes aquático e terrestre, na mata atlântica, Reserva Biológica do Alto da Serra de Paranapiacaba, Santo André, SP. Tese de doutorado, Universidade Estadual Paulista, Rio Claro.

SCHOENLEIN-CRUSIUS, I.H. \& MILANEZ, A.I. 1989. Sucessão fúngica em folhas de Ficus microcarpa L.f. submersas no lago frontal situado no Parque Estadual das Fontes do Ipiranga, São Paulo, SP. Rev. Microbiol. 20: 95-101.

SCHOENLEIN-CRUSIUS, I.H. \& MILANEZ, A.I. 1990a. Aquatic Hyphomycetes in São Paulo State, Brazil. I. First observations. Hoehnea 17:111-115.

SCHOENLEIN-CRUSIUS, I.H. \& MILANEZ, A.I. 1990 b. Hyphomycetes aquáticos no estado de São Paulo, Brasil. Rev. Microbiol. 13:61-68.

SCHOENLEIN-CRUSIUS, I.H., PIRES, C.L.A. \& MILANEZ, I.A. 1990. Sucessão fúngica em folhas de Quercus robur L. (carvalho) submersas em um lago situado no município de Itapecerica da Serra, SP. Rev. Microbiol. 21:61-67.

SCHOENLEIN-CRUSIUS, I.H., PIRES-ZOTTARELLI, C.L. \& MILANEZ, A.I. 1992. Aquatic fungi in leaves submerged in a stream in the atlantic rainforest. Rev.Microbiol. 23: 167-171.

SCHOENLEIN-CRUSIUS, I.H. \& TAUK, S.M. 1991. Fungal succession on Ocotea pulchella (Nees) Mez leaves in decomposition on "cerrado" soil treated with vinasse. Rev. Microbiol. 22:179-183.

SETZER, J. 1966. Atlas climático e ecológico do Estado de São Paulo. CIBPU-CESP, São Paulo.

TRUFEM, S.F.B. \& VIRIATO, A. 1990. Fungos micorrízicos vesículo-arbusculares da Reserva Biológica do Alto da Serra de Paranapiacaba, SP, Brasil. Revta brasil. Bot. 13:4954.

WARCUP, J.H. 1950. The soil plate method for isolations of fungi from soil. Nature 166:117-118. 